\title{
Control of Partner Robots by the Brain-Computer interface system
}

\author{
MortezaShahedifar, MasoumNazari \\ MSc student of Department of Electrical and Computer, University of Tabriz \\ PhD, Department of Electrical and Computer, University of Tabriz
}

\begin{abstract}
Some of people because of illness or the other factors can't do their works and BCI developments help them considerably. Especially during the two recent decades, considerable developments have been done in BCI domain. BCI system provides the situations that a person can control the devices and protest, robot types and ... with his/her brain signals.Nowadays many of researchers and university professors are working on brain-computer interface to develop it. Various scientists from the 1970 decade started activities about the BCI domain. In that time, the BCI sensors set on mics and monkeys and after that on human.
\end{abstract}

Keywords: brain-computer interface, robot control, BCI

\section{Introduction}

When the BCIs are described, two topic will be entered then, invasive and non-invaisive methods. In first method the electrode implemented directly on brain and the signals are sampled. In second method the electrodes connected on the EEG hat or other procedures. In second method the body do not injured. However there are some flaws in these methods, the non-invaisive method is more popular because of its lower problems. BCI technology has not been accepted completely yet after the 40 years. The challenge in this field is about EEG signal obtaining. EEG is non-invaisive method contain the signals of neural activities and unknown amount of noise. therefore the raw signals need to be filtered. Some group are working on EEG filtering, the main approach is personal specific parameters that can increase the classification accuracy (CA). However, these methods caused to lost unknown amount of EEG signal information. Therefore, the selected frequency filtering is a great challenge against the BCI setting up.Suggested method in this article shows a movement towards the EEG without noise. it based on QM principles, generating a pdf of time variable signal that evolves under the SWE effect.

The main challenge in BCI system is the less bandwidth from the linking channels. This article tries to show the challenge of updating surounded environment and the control device changes via an dynamic interface desigining. This approach removes the bandwidth lower than BCI and improves the device linking speed somewhat and BCI operator can reach to the forecasted speed.

The main targets of this article are as below:

- Assessing the QM concepts and obtaining ideas for the QM principles as the EEG filtering method for BCI.QM can be considered as a mathematic approach that describes the particle behavior in microscopic size but it is conceptual in neural network quantum framework.

-Evaluating the pre-processing based on RQNN for the EEG raw signal filtering. SWE conjugation in neural network paradigm originates a neural network, called RQNN.

-RQNN uses the various properties of time, frequency and classification for the filtered EEG operation.

-Assessment of a post-processing method for the suitable opposition with vague classifier outputs in an experimental EEG. The approach is De-biasing and various thresholds.

\section{Brain and machine interface}

Brain-computer interface is a direct interface between the human brain and artificial system. The target is that the devices control occur without using the environmental neurons or muscles just by the brain. $\mathrm{BCI}$ is the Electrophysiological signals interface with a computer.

In real situation, the BCI just uses the brain signals and eye and muscles movement should be considered as noise or artificial.

\section{Electrode implementation in brain}

The mature human brain has about 100 billion neuron that are the electric activity responsible. These electrical activities are signals for an EEG for the BCI system navigation. 


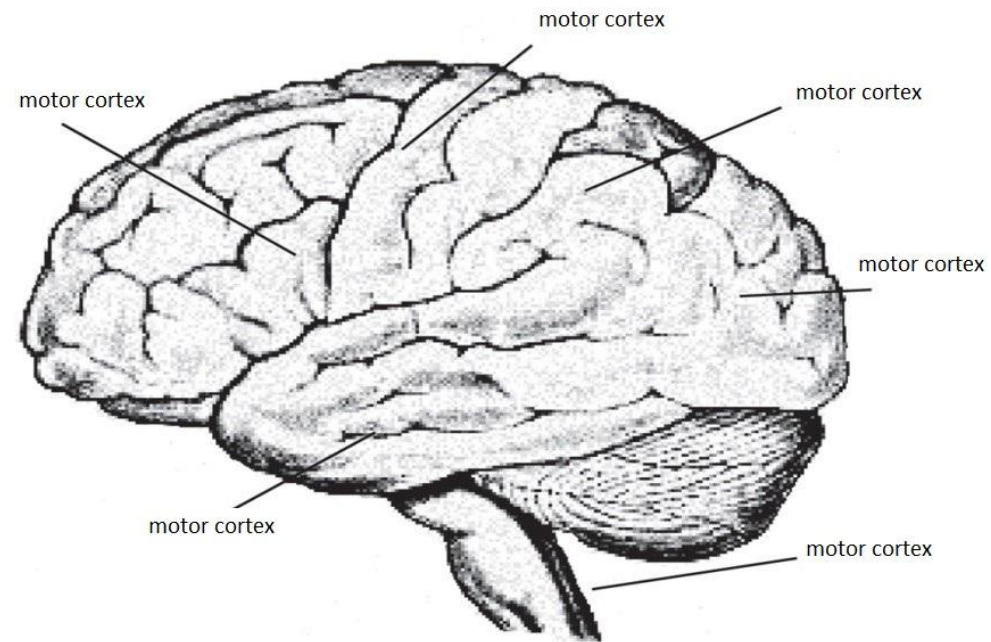

Fig1. A considerable review of some important parts of brain

The separated main parts of brain are Frontal lobe, the parietal, occipital and Temporal lobe.

The motor cortex locates behind the Frontal lobe and is the responsible of movement. Therefore, if the electrodes implemented along the motor cortex, the EEG signals along with movement images can be obtained. Thus, recognition of brain structure is crucial for the electrode implementation way on BCI operator head skin. If the optimum record sites link correctly to EEG, the BCI success probability will increase. The most of BCIs are invasive. Figure 2 shows the schematic image of BCI.

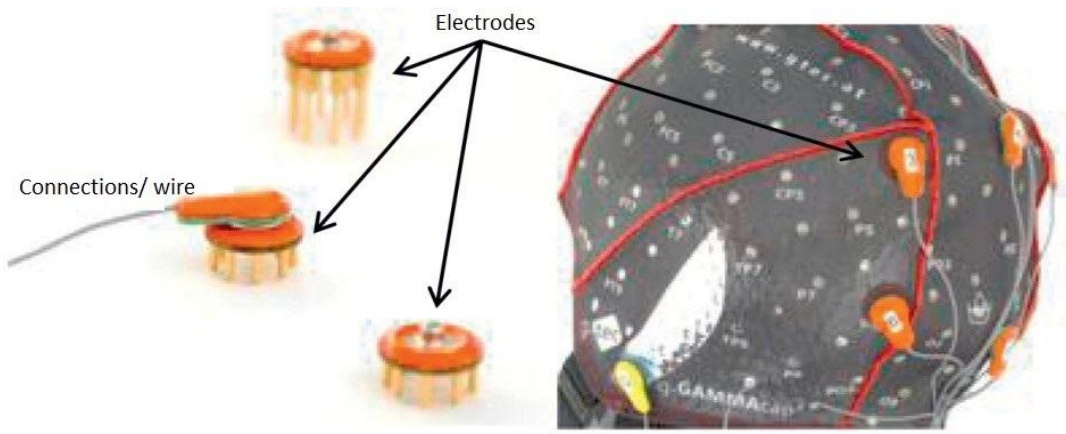

Fig2. Installation and setting up the EEG dry Hat.

\section{EEG wave rhythms}

Human brain generates continuously the electrical activities in voltage signal forms. The oscillation domain are $0.5 \mathrm{~Hz}$ to $100 \mathrm{~Hz}$ approximately. However, its general range is between 0.5 to $50 \mathrm{~Hz}$. This domain can be divided to different bands of frequency and depends on various recognition behaviors (table1). The $\Delta$ band is in 0.5 to $4 \mathrm{~Hz}$ and relates to deep sleep. This rhythm has the very slow waves but the highest domain. The $\theta$ band domain is in 4 to $7 \mathrm{~Hz}$ and is dominant in kids during the sleep or rest; in addition, it can be monitored in adults and adolescents during the drowsiness or the meditation state. The $\alpha$ band has the 8 to $13 \mathrm{~Hz}$ domain and generally is behind the head; it appears when a normal human closes his/her eyes. This wave is the popular rhythm for studying. $\mathrm{Mu}(\mu)$ is an especial type of $\alpha$ in $9-11 \mathrm{~Hz}$ domain and appears when the person is calm, thus this band can be used for the hand movement recognition. The $\beta$ has the $14-30 \mathrm{~Hz}$ and generally has the symmetric distribution in both sides of brain. Gamma $(\gamma)$ has the wide domain from 30 to $100 \mathrm{~Hz}$ and can be shown by the mutual sensory processing. Recent studies show that EEG recording suggested the $55-85 \mathrm{~Hz}$ Sensorimotor Rhythm (SMR). The gamma oscillation also has the casual effect on personal ability in BCI using. An important discussion is that each band share depends on especial situation for the total EEG curve. 
Table1. brain rhythms and details of relataed frequency band

\begin{tabular}{|c|c|c|c|}
\hline Details & $\begin{array}{l}\text { Natural } \\
\text { Domain } \\
(\mu \mathrm{V})\end{array}$ & $\begin{array}{l}\text { Frequency } \\
\text { Range }\end{array}$ & $\begin{array}{l}\text { Brain } \\
\text { Rhythm }\end{array}$ \\
\hline Mainly for the infants in deep sleep & $100>$ & $4-0 / 5$ & $\Delta$ \\
\hline $\begin{array}{r}\text { In kids and slowly in adults in frontal, cortex and } \\
\text { temporal. }\end{array}$ & $\begin{array}{c}100> \\
60-20\end{array}$ & $\begin{array}{c}7-4 \\
13-8\end{array}$ & $\theta$ \\
\hline $\begin{array}{l}\text { At brain warning time in adults, the most considerable } \\
\text { rhythms are in Occipital and cortex }\end{array}$ & $\begin{array}{l}50> \\
20>\end{array}$ & $\begin{array}{c}11-9 \\
30-14\end{array}$ & $\alpha$ \\
\hline $\begin{array}{r}\text { Frequency band related to hand movement, in sensory } \\
\text { motor cortex } \\
\text { Also relates to hand shaking } \\
\text { When the person concentrates or has some sensory } \\
\text { stimulations }\end{array}$ & $2>$ & $30<$ & $\mu$ \\
\hline
\end{tabular}

\section{The operation methods in BCI}

BCI systems can be classified in two state:

$\checkmark$ Simultaneous BCI that is computer-based and is upon to signs.

$\checkmark$ Asynchronous BCI that is operator-based and is not upon to signs

\section{Simultaneous BCI}

In Simultaneous BCI systems, the person operates after the visual receiving or hearing signs from the computer. The experiment can have two occurrence. Therefore, it is computer-based system and is upon to signs. Provided timing and controlling commands occurs by the computer, system and operator should be done based on computer timing commands. The BCI benefit is that a recognized brain activity is a setting upstimulator along with an especial sign. This caused that simultaneous BCI be the best selection for the most of BCI groups. A normal experiment of a BCI system takes time about 4 to 10 second and consists of MI duration, rest and inner duration.Million neuron's activity should be adapt to synchronize $\alpha$ rhythm that needs a period for developing. One another benefit of simultaneous BCI is that MI time is considered for the experiment rest time. Whereas it can be said that this system has some natural flaws. For example, the command can be sent just once in an experiment that limits the link bandwidth. Figure 3 shows a BCI paradigm. Training session contains the consequence but random iteration based on signs. This experiment starts with a constant cross that is shown in monitor center. After two second, a warning stimulus is presented as beep from. From second three to 4.25 a sign is shown, hinting the right or left on monitor. This person imagines the signs for 8 second. Moreover, the time between each experiment is about 0.5 to 2.5 . Sign random presentation shows the person concentration. $\mathrm{He} / \mathrm{she}$ needs four performances that each one consists of 40 experiment.

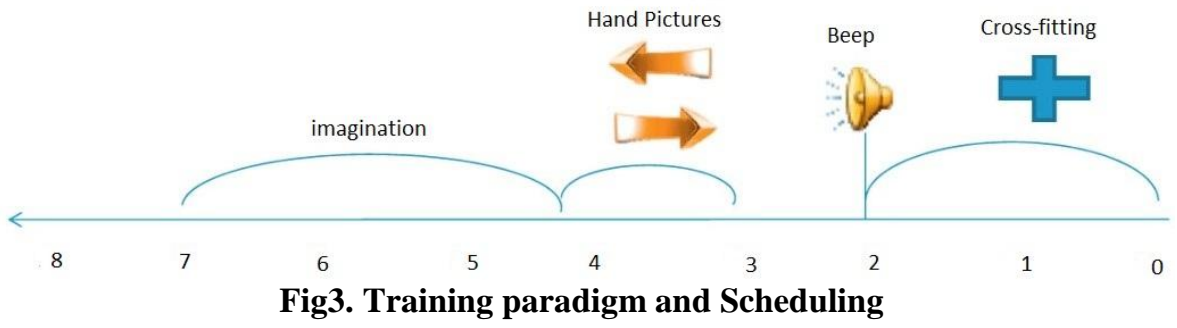

\section{Conclusion}

All methods and algorithms in this study based on assumed information via the literature review. This study talks about quantum concepts. For complex problem solving, the Recursive Quantum Neural Network (RQNN) can be used. In addition, it can be concluded that signal with low noise can be reached. Obtained results revealed that effective BCI system is an interface that transfers the information to BCI operator with high speed, reslution and accuracy. On the other hand the interface should adapt the signals among the brain and computer, decreasing the signal processin errors. Also it should be available in high similarity and diverse occurrences. 


\section{Refernces}

[1]. M. Tiefelsdorf, D.A. Griffith, Semiparametric filtering of spatial autocorrelation: the eigenvector approach, Environ. (2007).

[2]. J.R. Wolpaw, B. Niels, M. Dennis, G. Pfurtscheller, V. Theresa, Brain-computer interfaces for communication and control, Clin. Neurophysiol. (2002).

[3]. R.J. Croft, R.J. Barry, Removal of ocular artifact from the EEG: a re-view,Neurophysiol. Clin. (2000).

[4]. V. Gandhi, D. Coyle, G. Prasad, C. Bharti, L. Behera T. McGinnity M., Interfacing a dynamic interface paradigm for multiple target selection using a two class brain computer interface, in Indo - US Workshop on System of Systems Engineering, (2009).

[5]. V. Gandhi, G. Prasad, D. Coyle, L. Behera, T.M. McGinnity, An intel-ligent adaptive user interface (iAUI) for enhancing the communication in a brain-computer interface (BCI),Int. UKIERI Workshop on Fusion Brain-Comput. Interface Assist. (2011).

[6]. J.R. Wolpaw, B. Niels, M. Dennis, G. Pfurtscheller, V. Theresa, Brain-computer interfaces for communication and control, Clin. Neurophysiol. (2002).

[7]. M.A. Hofman, Evolution of the Primate Brain: From Neuron to Behavior, Elsevier Science, 2012.

[8]. J. Illes, B.J. Sahakian, The Oxford Handbook of Neuroethics, Oxford Univ Pr, 2011.

[9]. g.Tec,EEGand cap[Online].Available:,http://www.gtec.at/ProductsElectrodesand-Sensors/g.SAHARA-Specs Features.

[10]. T. Felzer, On the possibility of developing a brain-computer interface (BCI), technical University of Darmstadt, Darmstadt. (2001). 